\title{
A terminologia no estudo do usuário da informação
}

\author{
Ivette Kafure, Suzana Rocha, Vivianne Rodrigues, \\ Alessandra Souza, Karolina Bastos, Patrícia Raposo, \\ Tania Malheiros, Sonia Boeres, Ailton Feitosa
}

Universidade de Brasília - UnB, Brasil

\section{ARTIGO / ARTICLE}

\begin{abstract}
Resumo
Aborda o resultado de um estudo exploratório de conceitos/termos do Grupo de Pesquisa Informação, Design e Usabilidade (IDU), do Programa de Pós-Graduação em Ciência da Informação (PPGCinf), da Universidade de Brasília (UnB), na tentativa de definir uma nomenclatura interdisciplinar. A terminologia visa uma melhor comunicação, para o estudo do usuário da informação e a sua interação com os instrumentos tecnológicos na mediação e comunicação da informação. Apresenta terminologias utilizadas nos estudos de usuários da informação, com deficiência visual, da primeira infância e de interfaces de sistemas de informação. A pesquisa classifica-se de natureza aplicada, com abordagem qualitativa e em relação ao seu objetivo, exploratória. Conclui com uma identificação de uma terminologia comum dentro de um grupo interdisciplinar, que incorpora contribuições da Ciência da Informação, Artes, Comunicação, Psicologia Cognitiva, Educação, Computação e áreas afins, na busca de estratégias para uma melhor comunicação no exercício da pesquisa conjunta e colaborativa.
\end{abstract}

Palavras-chave

Estudo de Usuários da Informação ; Terminologia ; Usabilidade e Acessibilidade na Interação Humano-Computador

\section{The terminology in the study of user information}

\begin{abstract}
It an exploratory study of concepts/terms to define an interdisciplinary nomenclature of a research group of Information, Design, and Usability (IDU), of the Postgraduate Program of Information Science (PPGCinf), of the University of Brasilia (UnB). The IDU Group research through collaborative work about users and their interaction with interfaces for communicating and mediating the information. The proposal incorporates interdisciplinary contributions of Information Science, Arts, Design, Communication, Cognitive Psychology, Computer Science, Education and related areas. The terminology is intended to better communication for the user study of information and its interaction with the technological tools in mediation and communication of information. Presents terminology used in studies of information users, users with visual disabilities, early childhood and interfaces of information systems. Concludes with an identification of a common terminology within an interdisciplinary group, which incorporates contributions of Information Science, Arts, Communication, Cognitive Psychology, Education, Computer and related areas, in search of strategies for better communication in the course of joint and collaborative research.
\end{abstract}

Keywords

User Studies and Information Needs ; Terminology ; Usability and Accessibility in Human-Computer Interaction 


\section{Introdução}

Assim como os autores, que vêm de diferentes olhares, percursos e áreas de estudo, o trabalho a seguir é fruto da reflexão do Grupo de Pesquisa Informação, Design e Usabilidade (IDU), do Programa de Pós-Graduação em Ciência da Informação (PPGCinf), da Universidade de Brasília (UnB). O Grupo IDU tem como objetivo geral realizar pesquisas sobre os usuários e sua interação com as interfaces na comunicação e mediação da informação num trabalho colaborativo. Conta com um eclético grupo de colaboradores vindos de diferentes áreas, como a Ciência da Informação, Artes, Design, Comunicação, Psicologia Cognitiva, Computação, Educação e áreas afins. Este artigo se propõe a apresentar uma análise sobre a terminologia interdisciplinar no estudo do usuário da informação, se justifica na reunião da literatura sobre o assunto em relação aos estudos de usuários da informação, com deficiência visual, da primeira infância e de sistemas de informação, tendo sido criticamente analisada, sob diferentes perspectivas, dentro de cada área de estudo, e como método utilizou-se a revisão de literatura.

Para o processo de construção e desenvolvimento de grupos interdisciplinares dentro na Ciência da Informação, é importe o entendimento dos termos por seus membros. A interdisciplinaridade foi trazida para a Ciência da Informação pela própria multiplicidade da formação de todos os profissionais com ela envolvidos. O ARIST, em 37 anos de edição (1966-2003) publicou cinco artigos de revisão sobre história, teoria e epistemologia da Ciência da Informação. Pinheiro (2005, p. 16), a partir de interpretação epistemológico-histórica, dividiu as pesquisas sobre Ciência da Informação em três fases: de 1961 até 1969, chamando-a de reconhecimento do começo de um novo campo científico (Ciência da Informação), e discussões iniciais, principalmente sobre sua origem, denominação, conceitos, definições e natureza interdisciplinar; a segunda fase, de 1970 a 1989, de investigação de princípios, metodologia e teorias próprias, delimitando seu campo epistemológico, além de transformações advindas das novas tecnologias; e o último período, de 1990 em diante, na pesquisa de sua tese até 1995 e em artigo de 2005, de concretização de sua denominação, princípios, métodos e teorias, e debate sobre a sua natureza e relações interdisciplinares com outras áreas (BOERES, 2013).

Problemas complexos demandam enfoques interdisciplinares e soluções multidisciplinares. Entre os pioneiros havia engenheiros, bibliotecários, químicos, linguistas, filósofos, psicólogos, matemáticos, cientistas da computação, homens de negócios e outros vindos de diferentes profissões ou ciências (SARACEVIC, 1996, p. 48). Nem todas as disciplinas presentes na formação dessas pessoas tiveram uma contribuição igualmente acentuada, mas essa multiplicidade foi responsável pela introdução e permanência do objetivo interdisciplinar na Ciência da Informação.

Devido a essa interdisciplinaridade existe a necessidade definir uma terminologia para facilitar a comunicação e mediação da informação entre os vários pesquisadores, na aplicação de diferentes conhecimentos no estudo do usuário e a sua interação com o instrumento tecnológico.

No caso específico do Grupo IDU, para a definição da terminologia foram realizadas pesquisas bibliográficas e reuniões do grupo, às vezes com a presença de membros via teleconferência, e-mail e chat. Conseguir agrupar todos os membros para a definição de uma terminologia não é fácil, nem contínuo. Isto, pelas diferentes visões, comportamentos, interesses, ocupações e muitas vezes a distância na localização física.

\section{A terminologia}

A Terminologia é uma disciplina que estuda os conceitos e suas representações, abordando assuntos como natureza conceitual; relações conceituais; características dos conceitos; descrição dos conceitos; formação dos termos; padronização dos conceitos e termos; internacionalização dos conceitos. Em essência, os principais objetos de estudo da Terminologia são os conceitos, suas definições e os termos que os representam, inscritos em dado campo do conhecimento ou língua de especialidade.

Como ciência da área de Linguística, o domínio da Terminologia é a língua. Com o objetivo de garantir a comunicação, a língua utiliza palavras ou expressões como meio de representação dos conceitos. Conforme a atividade humana especializada, os conceitos representados por uma palavra podem variar em relação a contextos mais amplos. Esse fenômeno introduz a necessidade de que se definam as fronteiras entre a língua comum (língua geral), pertencente à sociedade tomada como um todo, e a língua de especialidade (ou linguagem especializada). Em um âmbito específico do conhecimento ou área profissional, a terminologia é o conjunto completo dos conceitos 
dessa área especializada e de suas definições, inserindo-se, assim, na linguagem especializada (léxico especializado). Os linguistas e os terminológos geralmente utilizam os termos língua de especialidade, linguagem especial, linguagem especializada, linguagem especialista ou linguagem para propósitos especiais, para designarem subsistemas léxicos cuja intenção é a comunicação não ambígua (WRIGHT; BUDIN, 1997, p. 330). Tal linguagem encontra-se na área da língua que aspira uma comunicação unívoca e livre de contradições em um domínio específico e cujo funcionamento encontra um suporte decisivo na terminologia estabelecida.

A leitura das definições de autores como autores como Felber (1984), Cabré (1993), Rondeau (1984) e Sager (1990), permite concluir que há três acepções para se definir Terminologia: a) Ciência Terminológica - campo inter e transdisciplinar que estuda os conceitos e suas representações; b) terminologia - agregado de termos que representa o sistema de conceitos de um domínio específico do conhecimento; c) terminologia - publicação na qual um sistema de conceitos de um campo de assunto específico é representado por termos. Em síntese, embora esses e outros autores como Heribert Picht, Alain Rey, Reiner Arntz, Pierre Auger, apresentem formulações diversas, depreende-se do seu pensamento que a Terminologia pode ser pensada em três sentidos: a) sentido científico (uma disciplina ou área do conhecimento); b) sentido técnico (um método de pesquisa); e c) sentido objetivo (um conjunto de termos).

A Terminologia, em sua essência, é uma ciência transdisciplinar, que possui interfaces com diversas áreas: Lingüística, Filosofia, Informática, Direito, Engenharia do Conhecimento, Ciência da Informação, entre outras. Na percepção Arntz e Picht (1995), existem três relações essenciais entre esta ciência e a Terminologia: a) a representação documentária é um campo especializado, com terminologia própria; b) a representação documentária utiliza fundamentos comuns à Ciência Terminológica; c) a representação documentária torna acessíveis os conhecimentos terminológicos, por meio da documentação terminológica. Assim, o trabalho terminológico é cada vez mais necessário para os diversos campos do saber, especialmente no que se refere a: a) organização do conhecimento (teoria geral da ciência, classificação dos conceitos para cada disciplina científica); b) transferência do conhecimento, habilidades especiais e tecnologias (educação e formação); c) tradução de textos científicos (tradução e interpretação); d) armazenamento e recuperação de informação especializada (linguagens de busca e recuperação, tesauros, índices, classificações) (GALINSKI; KROMMER-BENZ, 1988).

Levando em consideração a definição de terminologia e a problemática do usuário, a seguir, serão descritos pesquisas e conceitos relacionados ao estudo do usuário da informação, com deficiência visual, da primeira infância, do sistema de informação.

\section{0 usuário}

Guinchat e Menou (1994, p. 483) consideram que os usuários da informação podem ser divididos em três grupos principais: os usuários que ainda não estão na vida ativa profissional ou estudantes, os usuários engajados na vida ativa, cujas necessidades de informação se originam da sua vida profissional e o cidadão, cujas necessidades de informação são gerais e ligadas à sua vida social.

Segundo Figueiredo (1999, p. 79) "Estudo de usuários são investigações que se fazem para se saber o que os indivíduos precisam, em matéria de informação, ou então, para saber se as necessidades de informação, por parte dos usuários de um centro de informação estão sendo satisfeitas de maneira adequada". Para a autora, os estudos de usuários ressaltam os diversos canais de comunicação que se abrem entre os sistemas de informação e a comunidade a qual eles servem. Wilson-Davis (1977), ressaltava que um dos mais importantes desenvolvimentos da pesquisa no campo da informação e bibliotecas tinha sido a ênfase crescente atribuída aos estudos de usuários. Considerava dois tipos de abordagens para estudos de usuários, a quantitativa e a qualitativa, que os estudos de usuários podiam ser reunidos em dois grandes grupos: estudos centrados na biblioteca e estudos centrados no usuário. As necessidades de informação dos indivíduos e de seus grupos podem variar de acordo com as suas funções ou com os papéis que eles exercem no seu dia-a-dia, nesse sentido, Figueiredo (1999, p. 20) exemplifica esse modelo de variabilidade das necessidades e uso de informação segundo a atividade exercida pelo indivíduo (COSTA, SILVA E RAMALHO, 2009). 
Explicitado o que é usuário de informação na Ciência da Informação e suas necessidades, note-se que a interface do sistema

de informação ou instrumento tecnológico depende fortemente da compatibilidade (adequação) entre o modelo mental do usuário e a interface, quanto menor a compatibilidade entre a representação mental do usuário e a interface, menor será o entendimento da informação, e, portanto, menor a usabilidade e o design emocional da interface. Quando os usuários não entendem a ligação entre seu modelo mental, descrito neste contexto em termos de suas necessidades e expectativas (Quadro 1), e a interface do sistema de informação, a informação é dissipada, limitando, por conseguinte, a sua absorção.

\section{Quadro 1: modelo mental do usuário da informação}

\begin{tabular}{|l|l|}
\hline \multicolumn{2}{|c|}{ Modelo Mental } \\
\hline Necessidades & $\begin{array}{l}\text { busca de informação } \\
\text { acesso à informação } \\
\text { uso da informação }\end{array}$ \\
\hline Expectativas & $\begin{array}{l}\text { localizar rapidamente a informação procurada; } \\
\text { aprendizagem fácil da interface do sistema de informação; } \\
\\
\text { uso fácil da interface do sistema de informação; } \\
\text { satisfação na interação com o sistema de informação. }\end{array}$ \\
\hline
\end{tabular}

Fonte: Adaptado de Kafure, 2004, p. 4.

Para obter uma fundamentação teórico-metodológica sobre como o usuário se comunica, visualiza e entende a funcionalidade de um sistema de informação por meio da interface, foram estudados, entre outros, os princípios de: modelagem do usuário (modelo do Processador Humano - CARD; MORAN; NEWELL, 1983), modelagem da interação homem-computador (Teoria da Ação - NORMAN, 1983), modelagem da interface (modelo linguístico GREEN, 1995), modelo do processo de concepção do sistema (CLG - MORAN, 1981), modelagem de desempenho (GOMS - CARD; MORAN; NEWELL, 1983), guia ergonômico (SCAPIN, 1987).

Por outro lado, é preciso compreender os usuários, desde o ponto de vista emocional e cognitivo, como se comportam e recuperam a informação. Dessa maneira, Taylor (1968) identificou quatro níveis de necessidade de informação, correspondendo à sequência de quatro etapas de formulação da questão na recuperação da informação por parte do usuário: visceral, consciente, formalizada e comprometida. A necessidade visceral se define pela necessidade de informação real, mas não formulada. A necessidade consciente ocorre quando a pessoa a consegue descrever mentalmente. A necessidade formalizada, esta diz respeito a uma declaração formal da necessidade. Nesse estágio, o indivíduo já é capaz de fazer uma descrição racional da necessidade de informação, expressa, por exemplo, por meio de uma pergunta ou um tópico. Finalmente a necessidade comprometida representa a pergunta traduzida na linguagem do sistema. A questão formalizada é então modificada ou reelaborada numa forma que possa ser compreendida ou processada pelo sistema de informação. Concluída, a questão apresentada exprime a necessidade (EUCLIDES, 2007).

Os níveis identificados por Taylor (1968) podem ser relacionados aos três aspectos do design emocional sugeridos por Norman (2004): visceral, comportamental e reflexivo. O design visceral à necessidade visceral; o design comportamental à necessidade consciente desde ponto de vista objetivo e de usabilidade da interface; e, o design reflexivo à necessidade formalizada e comprometida da informação desde o ponto de vista subjetivo do usuário, à interpretação, à compreensão e ao raciocínio em relação à recuperação da informação por meio da interface. 
Nos anos 90, estudos científicos ilustram como a emoção e a cognição são absolutamente entrelaçadas, e, também, esclarecem que a emoção auxilia na escolha entre diversas opções e possibilidades em complemento com o conhecimento e a razão (DAMÁSIO, 1994, 2005). De acordo com Cañada (2005), está demonstrado que a percepção sobre um objeto muda, dependendo se ele é fácil de usar ou não, pois, segundo esse autor, nunca desenvolvemos vínculos emocionais positivos com algo cujo uso seja trabalhoso. Desta maneira, a relação entre emoção e usabilidade é direta. As emoções podem influenciar o processamento cognitivo, como a memória, a atenção, o processo de decisões e a resolução de problemas (LEDOUX, 2001).

Janczura (1999), em pesquisas de psicologia cognitiva, esclarece que os usuários da informação possuem diferenças que merecem ser estudadas com a finalidade de criar recursos eletrônicos e mecanismos para acessar a informação que sejam mais adaptados ao estilo de cada um na tarefa de acessar a informação, concluindo que:

- diferentes indivíduos procuram e processam a informação utilizando diferentes estratégias;

- diferentes estratégias podem ser mais ou menos efetivas para diferentes pessoas, em diferentes contextos;

- os indivíduos, em alguma medida, podem adotar consistentemente uma ou outra estratégia de processamento da informação (tais tendências são chamadas de estilos).

\section{Estudo de usuários}

O estudo de usuários na Ciência da Informação é de grande importância. As informações são organizadas para serem utilizadas por um usuário, e todos os serviços de informação existem para um determinado grupo de usuários. Daí a importância de se conhecer quem é esse usuário e quais são as suas necessidades de informação, como utilizam a informação e quais são seus comportamentos de busca. É por meio do "estudo de usuários" que se consegue chegar a esse conhecimento. Ele faz com que a informação chegue ao usuário certo, com rapidez e eficiência e em relação ao desenvolvimento dos acervos dos serviços de informações.

Gasque e Costa (2010) realizaram um levantamento quantitativo dos termos 'comportamento informacional', 'busca e uso da informação', 'busca da informação', 'uso da informação', 'necessidades de informação' e 'estudos de usuários' no catálogo on-line do IBICT (Instituto Brasileiro de Informação em Ciência e Tecnologia), nas bases LISA (Library and Information Science Abstracts), ERIC (Education Resource Information Center) e no buscador Scirus com o objetivo de conhecer o assunto. As buscas mostraram que o termo 'necessidades de informação' é o mais utilizado. O motivo pode estar relacionado à tradição, os primeiros estudos focavam-se nas necessidades dos usuários. O termo 'comportamento informacional', cunhado recentemente, começa a ser empregado, mas observase no Brasil que pesquisas intituladas Comportamento Informacional de Usuários, em geral ainda são conhecidas como "estudos de necessidades", e "estudos de usuários". Tanto Wilson (2000) como Pettigrew, Fidel e Bruce (2001) entendem o comportamento informacional como campo oriundo das limitações dos estudos de usuários, constituindo uma evolução teórico-metodológica dos estudos de comportamento informacional de usuários.

Historicamente, os métodos de estudo de usuários podem ser divididos em duas abordagens. Na primeira abordagem, a preocupação do pesquisador era centrada no serviço de informação, na investigação de técnicas e organização bibliográfica. Na segunda, com o passar do tempo, o enfoque dos estudos adotou um direcionamento mais voltado ao usuário. As suas necessidades de informação do usuário passaram a ter prevalência sobre os serviços, o que permitiu analisar e avaliar o comportamento dos usuários. A primeira abordagem, em que os estudos eram dirigidos aos sistemas de informação, é também conhecida como fase tradicional. A segunda, em que os estudos são direcionados às necessidades da pessoa, é denominada fase alternativa (CUNHA 1982; FIGUEIREDO, 1999; BAPTISTA e CUNHA, 2007).

Taylor $(1968,1982)$ sustenta a ideia de se focalizar, na percepção da utilidade e valor que o usuário traz para o sistema, nos estudos alternativos e em algumas questões do tipo: "que informação um indivíduo quer encontrar no 
sistema de informação? Que uso fará dela? Como o sistema pode melhor ser projetado para atender essas necessidades de informação?" (COSTA, SILVA e RAMALHO, 2009; COSTA e RAMALHO, 2010). Também Guinchat e Menou (1994), apontam a necessidade de se conhecer a fundo o usuário e também suas necessidades de busca e usos da informação.

O marco entre as abordagens tradicional e alternativa é muito bem definido por Dervin e Nilan (1986) que apontam a necessidade de mudança do foco do sistema para o usuário e também colocam a necessidade de conceituação e sistematização dessa área de estudos. Na verdade, já nas revisões sobre estudos de usuários realizados pela ARIST (Annual Review of Informations Science and Technology) a partir de 1966, começou-se a evidenciar as necessidades de busca e uso da informação. Essas revisões também mostraram a necessidade de sistematização de terminologias e conceituações para o estabelecimento de fundamentos bem definidos.

Dentro das abordagens alternativas de estudos de usuários podem ser encontradas as teorias e modelos de Taylor (1982), Belkin, Oddy e Brooks (1982), Dervin (1983), Kuhlthau (1999), Ellis (1989), Ellis, Cox e Hall (1993), Wilson (1981, 1994, 1997) e Choo (2003).

De acordo com Ferreira (1997) e Costa e Ramalho (2010) os estudos de usuários tem passado por diversas e diferentes fases, tais como: final da década de 1940, tinham como finalidade agilizar e aperfeiçoar serviços e produtos; na década de 50, intensificam-se no uso da informação; na década de 60, enfatizam o comportamento do usuário; na década de 70, passam a preocupar-se com mais propriedade com o usuário e a satisfação de suas necessidades de informação; na década de 80, estão voltados à avaliação de satisfação e desempenho; na década de 90, estão voltados ao comportamento informacional; $1^{\text {a }}$ década do século XXI, voltados tanto para o comportamento informacional, quanto para a avaliação de satisfação de desempenho.

Para o estudo do usuário ou um grupo de usuários específicos é preciso conhecer o seu perfil, o contexto ou instituição na qual utiliza o instrumento tecnológico, o seu modelo mental (Quadro, 1); a sua tarefa, isto é, o conhecimento que a pessoa tem acerca do que deve ser feito; a atividade, que é a realização da tarefa, o estado a alcançar; e, outras informações derivadas, como: a necessidade informacional, o comportamento informacional, preferências quanto a fontes de informação, tipos de suporte e/ou canais utilizados e como é a sua interação com outros usuários.

A tarefa pode ser descrita em vários níveis de abstração, desde a tarefa-objetivo (nível mais alto), passando pelas subtarefas (nível intermediário), até as mais simples, que podem ser descritas pelas ações elementares simples (nível inferior). Essa decomposição estrutural em vários níveis representam o plano idealizado pelo usuário para realizaram trabalho (KAFURE, 2004).

Segundo Baptista e Cunha (2007) a temática estudos de usuários vem sendo estudada há mais de 40 anos. Para se coletar dados sobre a atividade, têm sido utilizadas técnicas para detectar os dos passos que os usuários efetuam na realização de dada tarefa.

Os usuários da informação poderiam ser estudados a partir de alguns métodos, tais como:

- Formulários para questionário - identificação do perfil e de alguns tipos de necessidades como preferências quanto a suportes e materiais de pesquisa bem como assuntos de interesse.

- Observação - analisar o comportamento dos usuários a fim de detectar hábitos, rotinas e procedimentos com relação ao uso da informação e dos recursos informacionais.

- Roteiro estruturado de entrevista - verificar falhas, vantagens, problemas e facilidades do sistema de informação (não só tecnológico, considerar também os processos e fluxos de informação e como ela é disponibilizada). Provavelmente um diálogo poderia levantar fatores os quais ficariam limitados se utilizar um questionário.

- Observação o pesquisador capta a realidade a ser estudada, pode ser sistemática, não sistemática ou estruturada. 
- Análise de conteúdo busca determinar ênfase relativa ou a frequência ou de vários fenômeno na comunicação.

Provavelmente um único estudo, utilizando apenas um método, não possibilitaria ter acesso às várias facetas das necessidades de um grupo, inviabilizando a construção ou o aperfeiçoamento de um sistema/estrutura informacional. Dessa maneira, poderia ser apropriado a utilização de diversos métodos tanto para conhecer os usuários como para possibilitar a descoberta de diferentes aspectos quanto ao usuário e ao uso da informação.

É importante salientar que os estudos de usuários bem como a aplicação de adaptações para atender as necessidades e demandas detectadas, a fim de proporcionar um "ambiente informacional" adequado, devem considerar as condições financeiras, estruturais, políticas e interesses da instituição a qual esses pesquisadores estão inseridos. Afinal é a instituição que, muitas vezes, irá subsidiar de forma direta ou indireta os recursos informacionais e os aparatos necessários para essa estrutura.

\section{O usuário com deficiência visual}

O desafio da inclusão de pessoas com deficiência nos diferentes segmentos de relações sociais não é novo. A inclusão social tem uma série de indefinições, sendo vista de forma descontínua e assistemática por diferentes atores sociais (SENAC, 2002). Assim, discutir a questão terminológica é essencial, pois há várias denominações usadas quando nos dirigimos a pessoas com deficiência.

Sassaki (2005), em "Como chamar as pessoas que têm deficiência?" faz uma análise histórica dos termos usados ao se referir às pessoas que têm deficiência, no Brasil. Ele mostra que esses termos refletem os valores da sociedade da época. Segundo ele, durante séculos as pessoas que tinham deficiência eram chamadas de "inválidos" e considerados socialmente inúteis. No século XX, até meados dos anos 60 , o termo usado era "incapacitados", e de 1960 a 1980, passou a ser "defeituosos" (com deformidades), "deficientes" (indivíduos com alguma deficiência física, intelectual, auditiva, visual ou múltipla), ou "excepcionais” (indivíduos com deficiência intelectual). De 1981 até 1987, houve uma mudança do termo para "pessoas deficientes", e de 1988 até 1993, usavam-se os termos "pessoas portadoras de deficiência" ou "portadores de deficiência". A partir da década de 1990 surgem os termos "pessoas com necessidades especiais", "portadores de necessidades especiais", "pessoas especiais", "pessoas com deficiência", "portadores de direitos especiais".

Sassaki (2003) diz que o termo utilizado atualmente é "pessoa com deficiência". Segundo ele "no Brasil, tornou-se bastante popular, acentuadamente entre 1986 e 1996, o uso do termo portador de deficiência (e suas flexões no feminino e no plural)". No entanto, pessoas com deficiência alegam que a deficiência não é portada, como se fosse um objeto, mas faz parte da própria pessoa.

A Convenção sobre os Direitos das Pessoas com Deficiência da ONU adotou o termo "pessoas com deficiência" sendo, na atualidade, a linguagem mais apropriada para se referir a essas pessoas. No âmbito da legislação brasileira, a denominação "pessoas com deficiência" passou a ser o termo referendado pela Portaria n. 2.344 , de 3 de novembro de 2010. Essa portaria dá publicidade às alterações promovidas pela Resolução n. 1, de 15 de outubro de 2010, do CONADE em seu Regimento Interno, a começar pela substituição do termo "portador" para Pessoa com Deficiência (VARONOS, 2010).

Uma vez que o termo mais utilizado é "pessoa com deficiência", ele será o adotado neste trabalho, e nas citações serão mantidos os empregados pelos autores (Malheiros, 2013).

Assim como todas as pessoas, o usuário ou a pessoa com deficiência, tem uma grande necessidade de obter informações e realizar serviços na internet (KAFURE et al, 2012). No Modelo de Acessibilidade em Governo Eletrônico (gov.br e-mag, versão 3) afirmasse que em 2 de dezembro de 2004 foi publicado o decreto ํㅜ 5.296, de 2 que regulamenta a Lei no 10.048, de 8 de novembro de 2000, e o decreto oㅜ 10.098, de 19 de dezembro de 2000 . Essas normas, respectivamente, estabelecem critérios de atendimento para pessoas com deficiência e normas gerais e critérios básicos para a promoção da acessibilidade das pessoas portadoras de deficiência ou com mobilidade reduzida (Brasil, 2004). Também no capítulo VI desse decreto é descrito sobre o acesso à informação e a comunicação, que afirma no Art. 47, que no prazo de até doze meses a contar da data de publicação deste 
Decreto, será obrigatória a acessibilidade nos portais e sítios eletrônicos da administração pública na rede mundial de computadores (internet), para o uso das pessoas com deficiência visual, garantindo-lhes o pleno acesso às informações disponíveis.

Mas, muitas vezes, a forma de desenvolvimento do site impossibilita o acesso para estes usuários devido ao uso de tecnologias inadequadas.

Entre os serviços mais realizados por usuários com deficiência, estão: busca de informações de legislação que Ihes favoreça; acessos bancários; pagamentos de impostos; educação em geral. No entanto, grande parte dos sites que oferecem esses serviços não é desenvolvida obedecendo a padrões de acessibilidade, o que dificulta o acesso para esse público.

Para que 45 milhões de pessoas com deficiência, como indicado no censo IBGE de 2010, acessem os conteúdos e informações e se beneficie dos serviços ofertados pelo governo é necessário que as páginas sejam construídas de forma acessível, utilizando, além dos padrões de desenvolvimento web ou web standards, as diretrizes de acessibilidade. Entre as diretrizes de acessibilidade encontra-se as Diretivas para a Acessibilidade do Conteúdo da Web que estão em conformidade com as especificações da W3C.

O Consórcio World Wide Web (W3C) engloba centenas de empresas, órgãos governamentais e organizações independentes, além de uma equipe em tempo integral trabalhando para desenvolver estratégias, guias e recursos para acessibilidade à Web, visando ao crescimento democrático do seu acesso e uso. Por sua vez, os documentos das WCAG visam explicar como produzir conteúdos web (textos, imagens, formulários, sons e correlatos) acessíveis [às pessoas com deficiência] (W3C, 2010 apud COSTA; SILVA; RAMALHO, 2010, p. 139).

É importante destacar a falta de acessibilidade não decorrem da falta de políticas públicas. Há diversos projetos que recomendam e incentivam que os sites sejam acessíveis. Um exemplo é o Projeto Acessibilidade Virtual, um Módulo vinculado à Rede Nacional de Pesquisa e Inovação em Tecnologias Digitais (RENAPI), que busca fomentar a prática de pesquisa, ensino e extensão na Rede Federal de Educação Tecnológica (SETEC/MEC). Esse projeto visa desenvolver e disponibilizar soluções acessíveis, provendo acesso à maior gama possível de usuários, independente de limitação, situação ou tecnologia utilizada (MPOG, 2011).

A intenção é certificar que todos os produtos gerados no âmbito da RENAPI, adotem os seguintes conceitos de qualidade de uso de sistemas:

- acessibilidade: possibilidade de acesso a todos, independente de limitação (física, sensorial, cognitiva), situação ou tecnologia;

- usabilidade: facilidade de acesso a todos. Mede a produtividade, eficiência de uso e funcionalidade do ambiente;

- comunicabilidade: comunicação com todos. Diz respeito à capacidade de comunicar com clareza o conteúdo pretendido. Para atingir esses conceitos pautamos nossas atividades de acordo com os padrões de desenvolvimento com as diretrizes de acessibilidade, além de realizar as chamadas "Validação Automática" e "Validação Manual" com usuários com necessidades especiais, fato que confere autenticidade aos trabalhos desenvolvidos.

No caso da pessoa com deficiência visual, o estudo de usuários possui um aspecto peculiar, haja vista que para a pessoa com deficiência visual a apreensão do mundo ao seu redor deve se dar por meio de formas de viver sem ver, referindo-se neste caso à pessoa que tem perda total da visão. Pode referir-se também à pessoa que possui baixa visão, cuja apreensão do mundo por meio da visão possa ser dificultada. A classificação da redução da visão na baixa visão se dá nos níveis leve, moderada, severa, profunda. 
Assim, sua organização mental poderá focar-se em outros sentidos para descobrir o ambiente externo, podendo ser orientado pelas formas táteis, térmicas, olfativas, auditivas e cinestésicas (MASINI, 1991).

As necessidades de informação da pessoa com deficiência visual "seguem o padrão dos demais usuários, o que diferencia é o suporte onde é depositada a informação e um atendimento especial em relação ao acesso a essas informações. A diferença está na busca (falta de acessibilidade) e no acesso" (MALHEIROS, 2009, p. 45).

Nas últimas décadas, com o avanço tecnológico, com a inserção do uso do computador e outros aparatos tecnológicos no cotidiano dos indivíduos e com a propagação da Internet abriu-se maiores oportunidades para o armazenamento, disseminação e o provável uso da informação. Ou seja,

\begin{abstract}
o acesso às [Tecnologia da Informação e Comunicação] TICs se refere a uma série de possibilidades alternativas de acesso inclusivo à informação mediante tipos de hardware e software desenhados em sintonia com o conceito de desenho universal e ergonomia. Ao favorecer a biodiversidade humana natural, o desenho universal - como conjunto de conhecimentos que visam à concepção de produtos utilizáveis com eficácia, segurança e conforto - contribui para a melhoria da qualidade de uso de sistemas e da qualidade de vida como um todo. Ajustando-se o desenho universal à ergonomia, a última vem a ultrapassar o conceito de maior adaptabilidade à anatomia do ser humano (CORREIA; CORREIA; FRASSINETTI, 2009 apud COSTA; SILVA; RAMALHO, 2010, p. 138)
\end{abstract}

Nessas situações, para suprir as necessidades de informação da pessoa com deficiência visual, deve haver o fornecimento de dispositivos que possam ser adaptados a outros equipamentos tecnológicos. Visto que "a evolução natural dos modelos de interface com o usuário ocorrida nas últimas décadas popularizou o padrão baseado em metáforas puramente visuais. Este processo impediu o acesso de deficientes visuais a computadores e as novas tecnologias" (CARNEIRO, 2003, p. 5). Assim para que se tenha efetivo acesso a informação, é necessário haver neste caso o uso da tecnologia assistiva.

De acordo com o Instituto Nacional para a Reabilitação (INR) em seu Catálogo Nacional de Ajudas Técnicas (CNAT, 2008), a tecnologia assistiva, também

Todavia segundo Carneiro (2003, p. 5), "a evolução natural dos modelos de interface com o usuário ocorrida nas últimas décadas popularizou o padrão baseado em metáforas puramente visuais. Este processo impediu o acesso de deficientes visuais a computadores e as novas tecnologias".

Nessas situações, para suprir as necessidades de informação da pessoa com deficiência visual, deve haver o fornecimento de dispositivos que possam ser adaptados a outros equipamentos tecnológicos para que se tenha efetivo acesso a informação, havendo neste caso o uso da tecnologia assistiva.

De acordo com o Instituto Nacional para a Reabilitação (INR) em seu Catálogo Nacional de Ajudas Técnicas (CNAT, 2008), a tecnologia assistiva, também designada de ajudas técnicas, é definida como "qualquer produto, instrumento, estratégia, serviço e prática, utilizado por pessoas com deficiência".

Assim, há diversos dispositivos de acesso à informação os quais a pessoa com deficiência visual pode adaptar em seu computador e seus periféricos para possibilitar pleno acesso à informação e suprir uma necessidade de informação. Conforme Carvalho (2003) dentre esses dispositivos podem-se citar:

Geradores de informação visual ampliada

- Ampliadores de tela de computador;

- $\quad$ Sistemas de Circuito Fechado de Televisão (CFTV);

- Lentes ou sistema de lentes. 


\section{Geradores de informação auditiva}

- Braille falado;

- Sintetizador de voz.

\section{Geradores de informação tátil}

- Impressoras Braille;

- Regletes;

- Terminais de acesso em Braille para computadores;

- Copiadora em alto-relevo.

Transcritores

- Leitores de tela de computador;

- Sistemas de reconhecimento óptico de caracteres (OCR);

- Reconhecedores de voz;

- Transcritores Braille;

- Sistemas de reconhecimento de óptico de braille (OBR).

De tal modo, para que a necessidade de informação de um usuário seja atendida adequadamente "toda e qualquer informação deve ser orientada de acordo com o seu perfil, levando em consideração suas limitações, suas necessidades e o seu provável uso" (CASELLI, 2007, p. 11). Visto que "TODOS apresentamos algum tipo de desajustamento em relação à norma, em termos sociais, motores, do tipo de inteligência predominante, dos estilos de aprendizagem preferenciais, das capacidades preponderantes" (CORREIA; CORREIA; FRASSINETTI, 2013, grifo do autor).

Para que a necessidade de informação de um usuário seja atendida adequadamente é necessário adequar os recursos informacionais e da tecnologia ao atendimento dos usuários, preocupando-se em ouvi-los permanentemente (GARCEZ; RADOS, 2002). Ou seja, o estudo do uso da informação e da necessidade da informação deve-se voltar para a questão da inclusão, da acessibilidade,

[...] associando, para tanto, as questões para quem se destina a informação à para que se destina a informação. Pois o para quem, aqui, referir-se-á não só aos diversos papéis desempenhados pelos usuários, mas também aos seus diversos contextos e necessidades, por vezes "especiais" (de aprendizagem, de mobilidade, de comunicação, etc.), intervenientes no acesso à informação (COSTA; SILVA; RAMALHO, p. 137).

Dessa forma, um estudo de usuário voltado para pessoa com deficiência visual deve ser orientado tendo como abordagem de estudos o próprio usuário da informação, de forma que seja verificado se os sistemas de informação estão Ihe atendendo e como eles efetivamente o ajudam. Ferreira (1997) define essa abordagem aplicada aos estudos de usuário com a denominação de abordagem alternativa, na qual o usuário é visto como componente central dos sistemas de informação, sendo necessário obter "um conhecimento minucioso e preciso sobre o mesmo e sobre suas necessidades de buscas e usos da informação" (GUINCHAT, MENOU, 1994). 


\section{O usuário da primeira infância}

A relevância em estudar os usuários da primeira infância e os nativos digitais se deve ao fato do precoce contato com as tecnologias da informação. Segundo pesquisa do Comitê Gestor de Internet no Brasil (CBI.br) em 2010, crianças entre 5 a 9 anos já utilizavam jogos online na internet (90\%), seguindo por procura de material didático escolar (45\%) e acesso a mídias de massa buscando assim informação e entretenimento (34\%). A pesquisa revela que estes itens são o canal de contato das crianças para as atividades na Internet.

A partir do nascimento até os seis anos de vida da criança, é o período que compreende a primeira infância. Esse período é determinante para o desenvolvimento total da criança. Tapscott $(2010$, p.122) menciona que [...] "o cérebro é especialmente adaptável a influências externas nos primeiros três anos de vida" [...] nessa etapa e de acordo com os autores Aguiar, Nascimento e Barker (2007) "[...] a criança desenvolve grande parte do potencial cognitivo que terá quando adulto [...]" dos três aos seis anos de vida.

Entre dois e três anos, a criança fica mais a vontade e segura quanto ao equilíbrio (anda, corre, pula e sobe nos objetos) e coordenação motora (segura os objetos, movimenta-os com mais facilidade), e com o passar dos anos os sentidos vão aumentando tornando ainda mais fácil de modo geral todo o aprendizado ao longo de sua vida. Em seu trabalho sobre a "Epistemologia Genética" Piaget produz uma das mais importantes teorias do desenvolvimento humano. A teoria se divide em períodos, de acordo com as faixas etárias do desenvolvimento mental de cada indivíduo, que abordam desde o nascimento até a idade adulta.

Pádua (2009), citando Piaget, afirma que as crianças desde o seu nascimento até aproximadamente dois anos estão no estágio sensório-motor, as crianças dos dois aos seis anos encontram-se no estágio pré-operatório. Na fase sensório-motora a criança utiliza somente ações e percepções que "estimulam o desenvolvimento das estruturas mentais".

$\mathrm{Na}$ fase de estágio pré-operatório com aproximadamente dois anos é a fase da representação que "é a capacidade que a criança adquire, por meio das construções cognitivas, de pensar um objeto através de outro" (PÁDUA, 2009). $\mathrm{O}$ autor explica que Piaget chamou essa fase de estágio pré-operatório "porque significa que a criança utiliza a representação, mas ela tem todo um trabalho de assimilação, acomodação e equilibração de organizar essas representações num todo [...]." É a fase em que a criança apresenta grandes curiosidades sendo levantados por ela questionamentos, usando frequentemente a expressão "por que". Esses questionamentos favorecem de forma significativa o desenvolvimento cognitivo, linguístico (usa-se em média 3 a 4 palavras, tornando apta a conversar com pessoas mais velhas, fazendo uso de frases curtas) e intelectual da criança. O desenvolvimento intelectual abrange também a consciência da criança em que a própria se descobre como um ser e se descreve por meio de frases e gestos, tendo a memória e capacidade de concentração cada vez mais aguçada, compreendendo conceitos simples.

As crianças devem ser estimuladas, provocadas, chamadas para o aprendizado, mas não imposta a isso. Antunes (1998) "afirma que a prioridade para [...] aplicação [de estímulos] deve ser dada dos 2 aos 16 anos, período em que o cérebro produz uma substância chamada mielina, a qual envolve os neurônios, aumentando a velocidade nas informações", porém o "desenvolvimento da inteligência não se dá por acúmulos de informações, mas sobretudo por uma reorganização desta troca de inteligências, ou seja, crescer é uma forma de reorganizar a própria inteligência de forma a ter maiores possibilidades de assimilação" (PÁDUA, 2009).

As crianças tem um modo peculiar de aprender, elas aprendem brincando. Frobel (apud Kishimoto, 2008) elucida que "a brincadeira é importante para o desenvolvimento da criança, especialmente nos primeiros anos." [...] por meio da brincadeira a criança compreenderá o mundo e o representará, pois a criança possui a capacidade simbólica e cria significados a partir de objetos do seu mundo. Quando uma criança brinca, ela não quer saber das consequências da brincadeira ou do jogo, ela brinca, joga simplesmente pelo prazer de brincar e jogar, VíRGULA explorando tudo que lhe é desconhecido. No brincar a criança interage com tudo que está a sua volta e representa 0 que ela aprende. Pádua (2009, p.34) explica que "a necessidade de conhecimento do objeto pelo sujeito, leva-o a executar desde simples ações até operações sobre o objeto". Interação entre o sujeito e o objeto é que será produzido o conhecimento. 
As crianças do Terceiro Milênio nascem inseridas no mundo digital, apresentam habilidade e facilidade em lidar com aparatos tecnológicos. McLuhan (2002 apud FERREIRA, 2008) "a geração da infância do século XXI já nasce respirando tecnologia. A curiosidade e a espontaneidade naturais das crianças fazem com que elas se utilizem das tecnologias como extensões do corpo". O primeiro contato pode ocorrer em casa e ou na escola com uso de computadores, netbooks, notebooks, tablets, celulares e videogames. FERREIRA (2008, p. 2) "as crianças adquirem maior autonomia, uma vez que dominam o mundo digital de uma forma tão natural que causa espanto e estranhamento aos mais velhos". São os chamados "Nativos Digitais" que é uma terminologia utilizada por Marc Prensky (2001) para se referir a geração nascida depois dos anos 80. Conforme o autor salienta, os nativos são capazes de fazer várias coisas ao mesmo tempo como usar o celular, navegar na internet, ver TV e ouvir música. Esses sujeitos nasceram e cresceram com as tecnologias digitais e as usam brincando, não tendo medo.

Palfrey e Gasser (2011) definem os "Nativos Digitais" como pessoas nascidas na era digital (depois de 1980), que tem acesso às tecnologias digitais e as habilidades relacionadas com as mesmas. Os nativos digitais compartilhar uma cultura global, não rigidamente definida pela idade.

Além de brincar as crianças podem aprender dentro desse contexto tecnológico, o seu auto-aprendizado, pois ela não tem receios de explorar o que não conhecem, pelo contrário o novo a chama para se aventurar. Ferreira (2008, p.10) corrobora que "as tecnologias permitem que as crianças desenvolvam seu auto-aprendizado, visto que se aventuram, sem medo, por um ambiente livre que não lhes é nada estranho [...] a criança descobre o mundo sozinha escolhe informações [...] define as ações que condizem com suas necessidades". Necessidade que pode ser observada de acordo com o seu comportamento informacional.

Crespo e Caregnato (2003, p.249) observam que "comportamento de busca de informação acontece nos mais diversos contextos das vidas dos indivíduos". Kuhlthau (1991 apud CRESPO; CAREGANATO, 2003, p.250) afirma que "o processo de busca é centrado no indivíduo, [nesse caso, a criança] formando-se através da construção pessoal, na qual o usuário parte da informação para criar novos conhecimentos".

Desse modo, a sociedade tem se adaptado as novas maneiras de informação e comunicação de uma geração que pensa, aprende e se comunica velozmente. Tendo em consideração os dados referidos anteriormente surgem inquietações que precisam ser aprofundadas com pesquisas e embasamento teórico que fortalecerão a temática ainda pouco estudada na área da Ciência da Informação.

\section{O usuário na interação com o sistema de informação}

Os estudos de usabilidade para sistemas de informação automatizado têm crescido, o termo usabilidade substitui o termo user friendly, de conotação subjetiva. Os atributos ergonômicos do produto são a forma de medir usabilidade no que se refere a atitudes dos usuários e como eles interagem com o produto e sua aceitação. (Baptista e Cunha, p. 176)

O objetivo fundamental do desenvolvimento de interfaces que permitam a interação de humanos com equipamentos eletrônicos, sejam computadores, dispositivos móveis, televisores ou outras tecnologias, é a comunicação. Em especial, o desenvolvimento de aplicativos para computadores ou dispositivos móveis permite que pessoas comuniquem-se por diferentes vias, trocando informações compiladas, como notícias de jornais ou dados de clima e de tempo; enviando ou recebendo mensagens de correio eletrônico; utilizando recursos de localização geográfica; trocando mensagens de texto, áudio e vídeo; entretendo-se com jogos e passatempos; ou utilizando-se de outras inúmeras possibilidades.

Em qualquer das situações mencionadas, haverá sempre uma fonte de informação que pode ser uma pessoa, um banco de dados, ou qualquer outro repositório. Haverá, também, um destinatário que, do mesmo modo, poderá ser qualquer um desses agentes. O fato indiscutível é que seja qual for o momento: o de emissão da informação ou o de sua recepção, sempre será exigido o envolvimento de uma interação humana. Dito de outro modo, o objetivo das aplicações de software é a comunicação com os humanos. Ou, em um linguajar mais tecnicista: importa a comunicação com os "usuários". Mas, afinal, do ponto de vista da Comunicação Organizacional, qual é a definição de usuário? 
Em Comunicação Organizacional, as pessoas ou entidades que interagem com determinada instituição ou sistema de informações são comumente denominadas públicos. Entre outras atividades, a Comunicação Organizacional centra esforços no estudo e na proposição de estratégias que visam ao fortalecimento e à consolidação dos relacionamentos de instituições com os seus diferentes públicos. Obviamente, para que uma organização seja capaz de formular estratégias de relacionamento, necessita primeiro compreender as características e os interesses desses públicos - sejam pessoas, instituições ou grupos - e também de que modo tais públicos a influenciam, ou influenciam as suas atividades.

Diversos autores dedicaram-se à formulação de definições para a expressão públicos, bem como à proposição de metodologias que permitam às organizações identificarem-nos. O que há de mais comum entre os diferentes pontos de vista é a compreensão de que não há como definir a palavra público sem considerar a complexidade dos diversos enfoques culturais, étnicos, religiosos, geográficos, políticos e econômicos que a envolvem.

Outro ponto de convergência entre os autores é que os diferentes indivíduos ou grupos que se relacionam com cada organização o fazem de maneiras diferentes. Isso implica dizer que não há como restringir a definição de público a apenas uma pessoa ou ampliá-la a uma multidão. Ao empenhar-se na tarefa de identificar e descrever seus diversos públicos, a organização deve responder a questões como: de onde vem o público; como se forma; como se desenvolve; quais são suas variedades; quais são seus interesses; como se comporta; a que grupo social pertence; com é afetado (ou afeta) direta ou indiretamente pela atividade da organização.

Com essa preocupação em foco, as organizações que produzem sistemas informatizados ou web sites têm envidado esforços em busca de ofertar a cada público as informações segmentadas e atinentes aos seus interesses específicos. Em alguns segmentos como o bancário, por exemplo, é comum encontrarem-se sites que apresentam informações categorizadas em "para você", "para a sua empresa", "para o governo", entre outras categorias. Tem sido também mais comum o uso de metodologias como a modelagem de personas, para o desenvolvimento da arquitetura da informação em sistemas web. Resumidamente falando, a modelagem de personas consiste na técnica de se utilizar uma ficha descritiva de características pessoais de um personagem fictício, que pertence a um "tipo de pessoa" e que está ligado a algum segmento no universo dos públicos de uma instituição. Essa ficha, em geral, contém também dados demográficos daquele tipo de pessoa e alguns aspectos psicológicos ou sociais do seu relacionamento com a instituição.

Retomando a questão formulada anteriormente, do ponto de vista da Comunicação Organizacional, pode-se dizer que a definição de usuário, quando no contexto de interação com a interface de um sistema informatizado, confunde-se com a de definição de público: qualquer pessoa que individualmente, ou organizada em um grupo, se considera afetada direta ou indiretamente pela atividade real ou presumida de uma instituição. Admitindo-se tal definição, depreende-se, por extensão, que ao interagir com um sistema informatizado, um usuário poderá estar, por consequência, buscando relacionar-se com a instituição que produziu tal sistema.

Os usuários são a principal fonte de informação num estudo de usuários. De acordo com Miller (1992) e Cunha (2003), 85\% da informação útil é verbal e informal, antes que as fontes bibliográficas sejam consultadas, daí a importância do contato direto com as pessoas que vão interagir com o sistema de informação. Sugere-se que as instituições, ao buscarem as melhores práticas quanto ao desenvolvimento de interfaces com o usuário, incluam o estudo dos relacionamentos e das características dos seus diferentes públicos, como tema imprescindível ao sucesso de suas iniciativas.

\section{Considerações finais}

O grupo de pesquisa Informação, Design e Usabilidade (IDU) investiga o estudo de usuários e sua interação com as interfaces e a comunicação e mediação da informação. Sendo composto por uma equipe interdisciplinar que realiza pesquisas com o propósito de agregar conhecimento e experiências dentro e fora do grupo. Isto é para a sociedade mesma.

O trabalho colaborativo é importante e deve ser entendido e fomentado. 
A definição da terminologia entre os membros da equipe interdisciplinar tornou-se importante, para entender que há uma necessidade de identificar os termos e conceitos utilizados dentro da Ciência da Informação, no Grupo de Pesquisa e nos diferentes contextos que possam afetar os usuários e as pesquisas relacionadas aos mesmos.

Esta pesquisa em andamento ilustra a importância do trabalho terminológico. E, como cada vez mais, é necessário para os diversos campos do saber.

Levando em consideração que a terminologia é o conjunto completo dos conceitos utilizados de maneira especializada, no caso específico das pesquisas realizadas pelo Grupo IDU, no estudo do usuário da informação, com deficiência visual, da primeira infância e do sistema de informação. Foi possível identificar, que:

- $\quad$ o perfil do usuário precisa ser identificado desde o ponto de vista emocional e cognitivo;

- o termo 'necessidades de informação' é o mais utilizado nos estudos de usuários da informação;

- o termo mais utilizado é "pessoa com deficiência" para chamar as pessoas que têm deficiência;

- $\quad$ a terminologia "Nativos Digitais" é utilizada para se referir a geração nascida depois dos anos 80 da primeira infância;

- a definição de usuário, quando no contexto de interação com a interface de um sistema informatizado, confunde-se com a de definição de público: qualquer pessoa que individualmente, ou organizada em um grupo, se considera afetada direta ou indiretamente pela atividade real ou presumida de uma instituição.

Ao se iniciar um estudo de usuário é preciso primeiro avaliar o tipo de usuário a ser estudado. A partir daí, então pode-se estudar o seu contexto e a terminologia adequada à pesquisa relacionado ao estudo.

Tratando-se de qualquer que seja o tipo de usuário, é necessário que o foco seja dirigido às suas necessidades e expectativas (Quadro 1). Sendo analisados tanto seu comportamento de busca e uso da informação, como o acesso às informações.

Os estudos de usuários devem ser direcionados à individualidade das pessoas, considerando as suas experiências, necessidades e expectativas, permitindo uma visão holística.

\section{Bibliografía}

AGUIAR, Gabriela Azevedo.; NASCIMENTO, Marcos; BARKER, Gary. Breve panorama sobre a primeira infância. 2007. Disponível em: < http://www. promundo.org.br/wp-content/uploads/2010/05/Panorama-PI-Introducao.pdf>. Acesso em: 29 abr. 2012.

ANNUAL REVIEW OF INFORMATION SCIENCE AND TECNOLOGY. Washington: American Society for Information Science and Technology, v. 1-, 1966-.

ANTUNES, Celso. As inteligências múltiplas e seus estímulos. Campinas: Papirus, 1998.

ARNTZ, R., PICHT, H. Introducción a la Terminología. Traducción del alemán, Amelia de Irazazábal et al. Fundación Germán Sánchez Ruipérez. Madrid: Ediciones Pirámide, 1995.

BAPTISTA, Sofia Galvão; CUNHA, Murilo Bastos da. Estudo de usuários: visão global dos métodos de coleta de dados. Perspect. ciênc. inf. [online]. 2007, vol.12, n.2, pp. 168-184. ISSN 1413-9936 < http://dx.doi.org/10.1590/S1413-99362007000200011>

BELKIN, N. J., ODDY, B. N., BROOKS, H. M. ASK for information retrieval: part I, background and theory. Journal of Documentation, v.38, n.2, june, 1982. 
BOERES, Sonia. Levantamento sobre aspectos históricos da Ciência da Informação: fatos, eventos, personagens e outros aspectos relacionados ao processo de institucionalização da Ciência da Informação, entre as décadas de 1950 e os dias atuais. Trabalho da disciplina Fundamentos para a Recuperação da Informação, FCI/UnB, 2013.

BORGES, Teresa Maria Machado. O construtivismo na educação infantil. In: desenvolvimento e educação. São Paulo: ática, 1994. p. 16-24.

. A criança em idade pré-escolar:

BRASIL. Presidência da República. Casa Civil, Subchefia para Assuntos Jurídicos. Decreto N. 5296 de 2 de dezembro de 2004. Brasília. República, Casa Civil, Subchefia para Assuntos Jurídicos. 2004. Disponível em: <http://www.planalto.gov.br/ccivil 03/ Ato2004-2006/2004/Decreto/D5296.htm>. Acesso em 9 dez. 2012.

Ministério do Planejamento, Orçamento e Gestão (MPOG). Secretaria de Logística e Tecnologia da Informação, 2011.

Portaria no 03, de 07 de maio de 2007. Institucionaliza o Modelo de Acessibilidade em Governo Eletrônico - e-MAG no âmbito do Sistema de

Administração dos Recursos de Informação e Informática - SISP. Diponivel em:

<http://emag.governoeletronico.gov.br/emag/emag-3.pdf>. Acesso em: 9 dez. 2012.

CABRÉ, M. T. La terminologia. Teoria, metodologia, aplicaciones. Trad, para o espanhol por Carlos Tebé. Barcelona: Editorial Antártida, Empúries, 1993.

CAÑADA, J. Entrevista sobre los paradigmas del diseño. In: Santiago de Chile, 2005. Diseño emocional: definición, metodología y aplicaciones.

CARD, S.; MORAN, T.; NEWELL, A. The Psychology of Human-Computer Interaction. Hillsdale, NJ: Lawrence Erlbaum Associates, 1983.

CARNEIRO, Marcelo. Interfaces assistidas para deficientes visuais utilizando dispositivos reativos e transformadas de distância. 2003. 162f. Tese (Doutorado em Informática) - Pontifícia Universidade Católica do Rio de Janeiro, Rio de Janeiro, 2003.

CASELLI, Brígida Carla Almeida. Acesso à informação digital por portadores de necessidades especiais visuais: estudo de caso no Telecentro Acessível de Taguatinga. 2007. 94f. Dissertação (Mestrado em Ciência da Informação) - Departamento de Ciência da Informação e Documentação, Universidade de Brasília, 2007.

CATÁLOGO nacional de ajudas técnicas. [S.I.], 2008. O Instituto Nacional para a Reabilitação, IP, INR é responsável pela administração dos conteúdos do catálogo, a UMIC fornece o alojamento dos servidores, e a plataforma tecnológica é da responsabilidade do INOV - Inesc Inovação. Disponível: <http://www.ajudastecnicas.gov.pt/about.jsp>. Acesso em 11 maio 2012.

$\mathrm{CHOO}, \mathrm{C}$. W. A organização do conhecimento: como as organizações usam a informaçãoo para criar significado, construir conhecimento e tornar decisões. São Paulo: Senac, 2003.

CORREIA, Secundino; CORREIA, Patrícia; FRASSINETTI, Ese de Paula. Acessibilidade e desenho universal. Disponível em: <http://atividades.imagina.pt/uploads/cnoti/PDF/desenho universal.pdf>. Acesso em: 19 jun. 2013.

COSTA, Luciana Ferreira da; RAMALHO, Francisca Arruda. A usabilidade nos estudos de uso da informação: em cena, usuários e sistemas interativos de informação. Perspectivas em Ciência da Informação, v. 15, n. 1, p. 92-117, jan./abr. 2010.

COSTA, Luciana Ferreira da; SILVA, Alan Curcino Pedreira da; RAMALHO, Francisca Arruda. Para além dos estudos de uso da informação arquivística: a questão da acessibilidade. Ci. Inf., Brasília, DF, v. 39 n. 2, p.129-143, maio/ago., 2010.

COSTA, Luciana Ferreira da; SILVA, Alan Curcino Pedreira da; RAMALHO, Francisca Arruda. (Re)visitando os estudos de usuário: entre a "tradição" e o "alternativo". DataGramaZero, Rio de Janeiro, v.10, n. 4, p. 1-12, ago. 2009.

CRESPO, Isabel Merlo; CAREGNATO, Sônia Elisa. Comportamento de busca de informação: uma comparação entre dois modelos. Revista Em Questão. Porto Alegre, v. 9, n. 2, p. 271-281, jul. /dez. 2003. Disponível em: <

http://www.revistas.univerciencia.org/index.php/revistaemquestao/article/viewArticle/3639>. Acesso em: 20 maio 2012.

CUNHA, Murilo Bastos da. Notas de aula da disciplina: Fontes para a Recuperação da Informação. Brasília: UnB/CID, 2003.

Metodologias para o estudo de usuários de informação científica e tecnológica. Revista de Biblioteconomia de Brasília, v. 10, n. 2, p. 5-19, jul./dez. 1982.

DAMÁSIO, A. R. Somos esclavos de las emociones y del entorno. El País [periódico], España, 21 oct. 2005.

Descartes' error: emotion, reason, and the human brain. New York: Grosset, Putnam, 1994. 
DERVIN, Brenda. An overview of sense-making research: Concepts, methods and results. Paper presented at the annual meeting of the International Communication Association, Dallas, TX, May, 1983.

DERVIN, Brenda; NILAN, M. Informations needs and uses. In: WILLIAMS, M. (Ed.). Annual Review of Informatio Science and Technology, v. 21, p.3-33, 1986.

DIAS, Cláudia Augusto. Comunicação científica. Disponível em: <http://www.reocities.com/claudiaad/comunica.pdf>. Acesso em: 11 jun. 2012.

ELLIS, D. The derivation of a behavioural model for information retrieval system design. Information Studies. Sheffield, University of Sheffield, 1987.

ELLIS, D.; COX, D.; HALL, K. A comparison of the information seeking patterns of researchers in the Physical and Social sciences, Journal of Documentation, v.49, n.4, p. 356-9, Dec, 1993.

EUCLIDES, M. L. Representação das necessidades de informação na organização da informação: uma análise dos modelos teóricos de busca. 2007. 112f. Dissertação (Mestrado em Ciência da Informação) - Faculdade de Filosofia e Ciências, Universidade Estadual Paulista, Marília, 2007.

FERREIRA, Sueli Mara S. P. Estudo de necessidades de informação: dos paradigmas tradicionais à abordagem sense-making. Porto Alegre: ABEBD, 1997.

FERREIRA, Mayra Fernanda. A (in)formação na cultura de mídia tecnológica. In: COLÓQUIO INTERNACIONAL SOBRE A ESCOLA LATINO-AMERICANA DE COMUNICAÇÃO, 12, 2008, São Paulo. Disponível em: < http://encipecom.metodista.br/mediawiki/index.php/A (in)forma\%C3\%A7\%C3\%A3o da inf\%C3\%A2ncia na cultura de m\%C3\%A Ddia tecnol\%C3\%B3gica> .Acesso em: 23 fev. 2012.

FELBER, Helmut. Terminology Manual. Paris: Unesco, 1984.

FIGUEIREDO, Nice Menezes de. Avaliação de coleções e estudo de usuários. Brasília: ABDF, 1999. 96 p.

. Estudos de uso e usuários da informação. Brasília: IBICT, 1994.

GALINSKI, Christian; KROMMER-BENZ, Magdalena. Actividades internacionales en terminología. Actas: primer simpósio latinoamericano de terminologia, del 4 al 8 de abril de 1988. Caracas, Venezuela, 1988.

GARCEZ, Eliane Maria Stuart; RADOS, Gregório Jean Varvakis. Necessidades e expectativas dos usuários na educação a distância: estudo preliminar junto ao Programa de Pós-Graduação em Engenharia de Produção da Universidade Federal de Santa Catarina. Ciência da Informação, Brasília, v. 31, n. 1, p. 13-26, jan./abr. 2002. Disponível em: $<$ <ttp://revista.ibict.br/ciinf/index.php/ciinf/article/viewArticle/166>. Acesso em: 11 maio 2012.

GASQUE, Kelley Cristine Gonçalves Dias; COSTA, Sely Maria de Souza. Evolução teórico-metodológica dos estudos de comportamento informacional de usuários. Ciência da Informação, V. 39, n. 1 (2010).

GREEN, M. W. The Design of User Graphical Interfaces. 1995. Ph. D. Thesis. Tech. Report CSRI-70, Computer Systems Research Institute, University of Toronto, Canada, April 1995.

GUINCHAT, Claire; MENOU, Michel. Introdução geral às ciências e técnicas da informação e documentação. Brasília: IBICT, 1994.

HAAN, Geert de. ETAG, a formal model of competence knowledge for user interface desing. Amsterdam: Vrije Universiteit, 2000. (Doctoral Thesis).

JANCZURA, G. A. Acessibilidade conceitual em tarefas de categorização e memória. Brasília: Universidade de Brasília, CNPq, 1999.

KAFURE, Ivette. Usabilidade da imagem na recuperação da informação no catálogo público de acesso em linha. Brasília: Universidade de Brasília, Departamento de Ciência da Informação e Documentação, 2004. 311 p. (Tese de Doutorado)

KAFURE, Ivette; ROCHA, Suzana. F. ; RODRIGUES, V. R. ; SOUZA, A. S. ; SILVA, Karolina Vieira da ; RAPOSO, Patrícia Neves ; MALHEIROS, T. M. C. ; CUNHA, M. B. ; CRUZ, E. S. ; BOERES, Sonia ; FEITOSA, A. L. G. ; Osandon, Michel ; MARTINEZ, W. F. R. ; SOUZA, R. R. . Terminologia interdisciplinar no estudo do usuário da informação. VIII Workshop Internacional em Ciência da Informação (WICl 2012), Brasília, 2012.

KUHLTHAU, C. C. Accommodating the User's Information Search Process: Challenges for Information Retrieval System Designers. Bulletin of the American Society for Information Science, Washington, v. 25, n. 3, p. 12-16, Feb./Mar. 1999. Disponível em: <http://www.asis.org/Bulletin/Feb-99/kuhlthau.html>. Acesso em: 15 jul. 2007. 
LE COADIC, Yves-François. A ciência da informação. 2. ed. Brasília: Briquet de Lemos Livros, 2004. 124 p.

LEDOUX, J. E. O Cérebro emocional: os misterios alicerces da vida emocional. Rio de Janeiro: Objetiva, 2001.

KISHIMOTO, Tizuko Morchida. O jogo e a educação infantil. In: KISHIMOTO, Tizuko Morchida (Org.). Jogo, brinquedo, brincadeira e a educação. 3.ed., São Paulo : Cortez, 1999. n. 1, p. 13-43.

MALHEIROS, Tania Milca de Carvalho. Estudo do usuário deficiente visual e subsídios para uma política de desenvolvimento de coleções da Biblioteca Central da Universidade de Brasília. 2009. 94f. Monografia (Especialização em Gestão Universitária) Faculdade de Economia, Administração, Contabilidade e Ciência da Informação e Documentação, Universidade de Brasília, Brasília, 2009.

MASINI, Elcie F. Salzano. Perceber e o relacionar-se do deficiente visual: orientando professores especializados(o). In: REUNIÃO ANUAL DA ASSOCIAÇÃO NACIONAL DE PÓS-GRADUAÇÃO E PESQUISA EM EDUCAÇÃO, 14., 1991, São Paulo. Anais... São Paulo: ANPED, 1991.

MATTAR, João. Games em educação: como os nativos digitais aprendem. São Paulo: Pearson Prentice, 2010.

MILLER, K. O Óleo de Lorenzo. Direção George Miller. Produção: Arnald Burk. Roteiro: Doug Mitchelle e George Miller. City studios: Universal pictures. Filme (1:34 min), 1992.

MORAN, T. The Command Language Grammar: a representation for the user interface of interactive computer systems. International Journal of Man-Machine Studies, v. 15, p. 3-50, 1981.

NORMAN, Donald A. Emotional Design: Why we love (or hate) everyday things. New York: Basic Books, 2004.

Cognitive engineering. In: NORMAN, D. A.; DRAPER, S. W. (Eds.). User centered system design: new perspectives on human-computer interaction. Hillsdale, NJ: Erlbaum Associates, 1986. 526 p.

Some observations on mental models. In: GENTNER, Dedre; STEVENS, Albert L. Mental Models. Hillsdale, NJ: Lawrence Erlbaum Associates, 1983. p. 7-14.

PÁDUA, Gelson Luiz Daldegan de. A epistemologia genética de Jean Piaget. Revista FACEVV. Vila Velha, n. 2, p. 22-35, 1 sem. 2009. Disponível em:

<http://prolicenmus.ufrgs.br/repositorio/moodle/material didatico/didatica musica/turma ef/un21/links/epistemologia genetica.pdf> Acesso em: 03 fev. 2012

PALFREY, John.; GASSER, Urs. Nascidos na era digital: entendendo a primeira geração dos nativos digitais. Tradução de Magda França Lopes. Porto Alegre: Artmed, 2011. p.324.

PINHEIRO, Lêna Vânia Ribeiro. Processo evolutivo e tendências contemporâneas da Ciência da Informação. Informacao \& Sociedade, v.15, n.1, p. 13-48, jan./jun. 2005. Disponível em: < http://www.ies.ufpb.br/ojs/index. php/ies/article/view/51 >. Acesso em: 12 de abril de 2013.

PRENSKY, Marc. Digital Natives, Digital Immigrants. MCB University Press, 2001. Disponível em: <http://www. marcprensky.com/writing/Prensky\%20\%20Digital \%20Natives,\%20Digital\%20Immigrants\%20\%20Part1.pdf>. Acessado em maio de 2012

RONDEAU, Guy. Introduction à la terminologie (2e édition). Québec: Gaétan Morin, 1984.

SAGER, Juan C. A Practical Course in Terminology Processing. Amsterdam: John Benjamins, 1990.

SARACEVIC, T. Ciência da Informação: origem, evolução e relações. Perspectivas em Ciência da Informação, v. 1, n.1, p. 41-62, jan./jun. 1996. Disponível em: <http://www.scribd.com/doc/51790111/SARACEVIC-T-Ciencia-da-Informacao-origem-evolucao-erelacoes-Perpectivas-em-Ciencia-da-Informacao-v-1-n-1-p-41-62-jan-jun-1996>. Acesso em: 12 de abril de 2013.

SCAPIN, D. L. Guide ergonomique de conception des interfaces homme-machine. France: INRIA, 1987. (Rapport INRIA n. 77).

TAPSCOTT, Don. O cérebro da geração internet. In: . . A hora da geração digital: como os jovens que cresceram usando a internet estão mudando tudo, das empresas aos governos. Tradução de Marcello Lino. Rio de Janeiro : Agir negócios, 2010. Cap. 04 , p. 121-148

TAYLOR, R. S. The process of asking questions. American Documentation, 13(4), 391-396, 1962.

TAYLOR, R. S. Question-Negotiation and Information Seeking in Libraries. College \& Research Libraries, 29 (3), 1968.

TAYLOR, R. S. Value-Added in the Information Life Cycle. Journal of the American Society for Information Science, 1982. 
WILSON-DAVIS, K. The Centre for research on users studies: ains and functions. Aslib Proceesdings, v.29, n.2, p.67-73, 1977.

WILSON, T. D. Information behaviour: an interdisciplinary perspective. Informatoin Processing and Management, 1997. 33(4): $\mathrm{p}$. 551-572.

Information needs and uses: fifty years of progress? In B. C. Vickery (Ed.), Fifo' years of information progress: a Journal of Documentation review (pp. 15-51). London, Aslib, 1994.

. On user studies and information needs. Journal of Documentation, 37(1), 3-15, 1981.

WRIGTH, S.E.; BUDIN, G. Handbook of Terminology Management. Amsterdam/Philadelphia: John Benjamins, The Netherlands/USA. Volume 1: 1997; Volume 2: 2001.

SENAC. Divisão Nacional. Deficiência e competência: programa de inclusão de pessoas portadoras de deficiência nas ações educacionais do Senac. Rio de Janeiro: Senac Nacional, 2002.

SASSAKI, Romeu Kasumi. Terminologia sobre deficiência na era da inclusão. In: VIVARTA, Veet (Coord.). Mídia e deficiência. Brasília: Andi/Fundação Banco do Brasil, 2003. p. 160-165.

Como chamar as pessoas que tem deficiência? São Paulo, 2005. Disponível em: <http://www.aderon.com.br/mznews/data/comochammar.pdf >. Acesso em: 5 fev. 2009.

MALHEIROS, Tania Milca de Carvalho. Necessidade de informação do usuário com deficiência visual: um estudo de caso da Biblioteca Digital e Sonora da Universidade de Brasília. Dissertação de Mestrado, Programa de Pós-Graduação em Ciência da Informação, Universidade de Brasília, Brasília, 2013.

VARONOS, Cybelle. Portaria n. 2.344, de 3 de novembro de 2010. Deficiente alerta.10 nov. 2010. Disponível em: <http://deficientealerta.blogspot.com.br/2010/11/portaria-n-2344-de-3-de-novembro-de.html>. Acesso em jun. 2012.

\section{Dados dos autores}

Ivette Kafure

Brasil. Grupo de Pesquisa Informação, Design e Usabilidade. Professora da Faculdade de Ciência da Informação da Universidade de Brasília - UnB.

Ivettek@unb.br

\section{Suzana Rocha}

Brasil. Grupo de Pesquisa Informação, Design e Usabilidade. Mestranda do Programa de Pós-Graduação em Ciência da Informação da Universidade de Brasília - UnB.

rocha.suzanafr@gmail.com

\section{Vivianne Rodrigues}

Brasil. Grupo de Pesquisa Informação, Design e Usabilidade. Mestranda do Programa de Pós-Graduação em Ciência da Informação da Universidade de Brasília - UnB.

\section{vivianne.darocha@gmail.com}

\section{Alessandra Souza}

Brasil. Grupo de Pesquisa Informação, Design e Usabilidade. Mestranda do Programa de Pós-Graduação em Ciência da Informação da Universidade de Brasília - UnB.

\section{alessandrabuba@gmail.com}

\section{Karolina Bastos}

Brasil. Grupo de Pesquisa Informação, Design e Usabilidade. Graduada em biblioteconomia pela Universidade de Brasília - UnB.

\section{karollinna@gmail.com}




\section{Biblíg A terminologia no estudo do usuário da informação}

\section{Patrícia Raposo}

Brasil. Grupo de Pesquisa Informação, Design e Usabilidade. Mestre em Educação pela Universidade de Brasília UnB.

\section{patricianraposo@gmail.com}

Tania Malheiros

Brasil. Grupo de Pesquisa Informação, Design e Usabilidade. Mestranda do Programa de Pós-Graduação em Ciência da Informação da Universidade de Brasília - UnB.

taniasaboia@yahoo.com.br

\section{Sonia Boeres}

Brasil. Grupo de Pesquisa Informação, Design e Usabilidade. Doutoranda do Programa de Pós-Graduação em Ciência da Informação da Universidade de Brasília - UnB.

\section{sonia.boeres@gmail.com}

\section{Ailton Feitosa}

Brasil. Brasil. Grupo de Pesquisa Informação, Design e Usabilidade. Possui Doutorado em Ciência da Informação pela Universidade de Brasília - UnB. Universidade de Brasília - UnB.

ailton.feitosa@gmail.com

$\begin{array}{ll}\text { Recebido- Received } & : 2013-04-07 \\ \text { Aceito-Accepted } & : 2013-06-30\end{array}$

(cc) EY New articles in this journal are licensed under a Creative Commons Attribution 3.0 United States License.

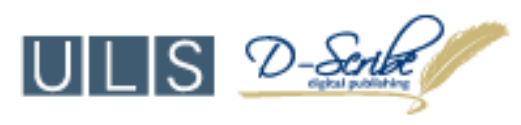

This journal is published by the University Library System of the University of Pittsburgh as part of its D-Scribe Digital Publishing Program and is cosponsored by the University of Pittsburgh Press. 